\title{
Elements regulating Potato leafroll virus sgRNA1 translation are located within the coding sequences of the coat protein and read-through domain
}

\author{
Adrianna Łoniewska-Lwowska* , Sylwia Chełstowska , Włodzimierz Zagórski-Ostoja \\ and Andrzej Pałucha ${ }^{\varpi}$ \\ Department of Protein Biosynthesis, Institute of Biochemistry and Biophysics PAS, Warszawa, Poland
}

Received: 23 March, 2009; revised: 11 August, 2009; accetpted: 09 September, 2009

available on-line: 16 October, 2009

\begin{abstract}
Translation of viral proteins from subgenomic RNAs (sgRNAs) is a common strategy among positive-stranded RNA viruses. Unlike host mRNA, sgRNA of Potato leafroll virus (PLRV) does not possess a cap at its $5^{\prime}$ end nor a poly(A) tail at the $3^{\prime}$ terminus, both of which are known to be crucial for translation of RNA in eukaryotic cells. Here, we demonstrate, that in wheat germ extract (WGE) truncation of the sgRNA1 $5^{\prime}$ UTR increases translation efficiency, as it has previously been observed in rabbit reticulocyte lysate (RRL), whereas removal of the $3^{\prime}$ UTR does not affect translation. We also describe two regulatory elements located within the coding sequence of the coat protein (CP) gene and its read-through domain (RTD) and are responsible for regulation of in vitro translation of the PLRV sgRNA1. The first element is composed of the purine sequence AAAGGAAA located between the AUG codons of the CP and 17K genes. Deletion of this domain or its substitution by pyrimidines reduced by half the translation of both genes, whereas deletion of the RTD resulted in a 3.6-fold reduction in translation efficiency. This is the first report of translation regulatory elements of plant viruses located within a coding region.
\end{abstract}

Keywords: polerovirus, subgenomic RNA, in vitro translation

\section{INTRODUCTION}

Potato leafroll virus (PLRV) is a type member of genus Polerovirus, family Luteoviridae. PLRV virions encapsidate a non-polyadenylated, single-stranded (ss) positive-sense RNA molecule of nearly $6 \mathrm{~kb}$ in length with a genome-linked protein (VPg) at the 5' end (Mayo et al., 1982). Analysis of the nucleotide sequence revealed the presence of eight ORFs organized in two gene clusters (Fig. 1). Those two clusters are separated by an intergenic region (Mayo et al., 1989; Mayo \& Ziegler-Graff, 1996). To express its genes PLRV employs a variety of mechanisms. The ORFs in the first cluster (ORF0, ORF1 and ORF2) are translated from the genomiclength RNA (gRNA), whereas ORFs designating the second cluster are expressed from sgRNA1 (ORF3, ORF4 and ORF5) and sgRNA2 (ORF6 and ORF7). The fact that PLRV sgRNA1 is an exact copy of genomic non-polyadenylated RNA poses the question of how this RNA substitutes for the major cytoplasmic functions associated with poly(A), i.e., control of mRNA stability and modulation of translation efficiency. Viruses that lack a cap and/ or a poly(A) tail must have developed alternative strategies for the regulation of their translation. Particular attention has been directed towards noncoding elements with respect to their influence on gene expression. Leader sequences (LS), also called 5' UTRs, have multiple effects on the translation of downstream-located genes and on mRNA stability (Gallie \& Walbot, 1992; Nicolaisen et al., 1992;

\footnotetext{
${ }^{\square}$ Corresponding author: Institute of Biochemistry and Biophysics PAS, A. Pawińskiego 5a, 02-106 Warszawa, Poland; phone: (48) 22592 3411; fax: (48) 22658 4636; e-mail: alfap@ibb.waw.pl

* Present affiliation: International Institute of Molecular and Cell Biology, Ks. Trojdena 4, 02-109, Warszawa, Poland

Present affiliation: National Medicines Institute, Chełmska 30/34, 00-725 Warszawa, Poland

Abbreviations: CP, coat protein; gRNA, genomic RNA; IRES, internal ribosome entry site; LS, leader sequence; ORF, open reading frame; RRL, rabbit reticulocyte lysate; RTD, read-through domain; PCR, polymerase chain reaction; PLRV, Potato leafroll virus; sgRNA, subgenomic RNA; UTR, untranslated region; WGE, wheat germ extract.
} 


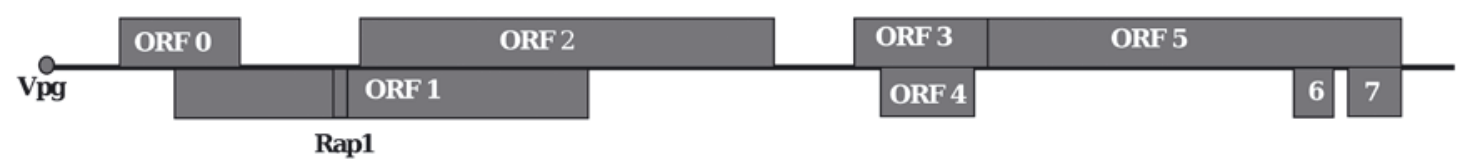

gRNA

$5^{\prime} \frac{1}{2}$

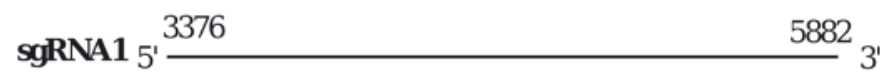

sgRNA2 $5, \frac{5190 \quad 5882}{5} 3^{\prime}$

Figure 1. Schematic representation of PLRV genome and viral RNAs of positive polarity.

ORF, open reading frame; 6, 7, ORF6 and ORF7; Vpg, viral protein genome-linked; Rap1, replication-associated protein 1. Nucleotide numbers according to the PLRV sequence (accession number X74789).

Pooggin \& Skryabin, 1992; Zelenina et al., 1992; van Vossen et al., 1993; Johnston \& Rochon, 1996; Turner et al., 1999). The 5' leaders of several plant viral mRNAs, including members of the tobamoviral, potyviral, comoviral and luteoviral families, are responsible for conferring cap-independent translation (reviewed by Kneller et al., 2006).

The role of the 3' UTR on translation efficiency and viral RNA stabilization has also been studied in detail in vitro and in vivo and demonstrated for human and animal viruses, such as $\mathrm{HCV}$ and rotavirus (Vende et al., 2000; Bradrick et al., 2006), as well as for plant viruses such as Tomato bushy stunt virus (TBSV), Satellite tobacco necrosis virus (STNV) and Turnip crinkle virus (TCV) (Danthinne et al., 1993; Wu \& White, 1999; Qu \& Morris, 2000). In the case of Barley yellow dwarf virus PAV isolate (BYDV-PAV) belonging to the genus Luteovirus of family Luteoviridae, a highly efficient cap-independent translation of gRNA and sgRNA1 is conferred by a 109-nt BYDV-PAV capindependent translation element (BTE) located in the 3' untranslated region (UTR) (Wang \& Miller, 1995; Wang et al., 1997; Guo et al., 2000). As it was shown in wheat germ extract (WGE) system, BTE mimics the $5^{\prime}$ cap and can be functionally replaced by a cap analogue, but not by the poly(A) tail (Wang et al., 1999). The presence of the above regulatory elements has also been proposed for other members of the genus Luteovirus, e.g., Bean leafroll virus (BLRV) (Domier et al., 2002).

For PLRV the implication of the non-coding regions as determinants of translation efficiency has also been investigated. The influence of both UTRs on protein expression of gRNA and sgRNAs has been demonstrated (Juszczuk et al., 1997; 2000). Analysis of protein expression in rabbit reticulocyte lysate (RRL) showed that the sgRNA1 LS appears to be a translation silencer of the genes located down- stream, and it was proposed that the role of the $5^{\prime}$ UTR is to maintain a proper ratio of the proteins synthesized. Furthermore, in vitro experiments revealed that, at least in RRL, there is no translation enhancer element within the $3^{\prime}$ UTR. However, it could not be excluded that the $3^{\prime}$ UTR-located elements of PLRV are not active in the experimental system used, as it was demonstrated for BYDV (PAV) (Wang \& Miller, 1995). This suggests that PLRV has elaborated as yet unknown mechanisms to modulate translation and RNA stability. Therefore, it seemed interesting and important to investigate the elements modulating the translation efficiency.

In this study we demonstrate that two regions of the coding sequence of the $\mathrm{CP}$ gene and its readthrough domain may modulate the translation from sgRNA1 in wheat germ extract.

\section{MATERIALS AND METHODS}

Templates for in vitro transcription. Two plasmids were used for amplification of PCR-generated templates for in vitro synthesis of RNA transcripts. Plasmid pJF, containing the full-length cDNA of the PLRV genome fused to the T7 RNA polymerase promoter (Sadowy et al., 1998) and psgRNA1, a pUC118 derivative, carrying viral cDNA corresponding to sgRNA1 (unpublished). All primers used for amplification of viral cDNA fragments are listed in Table 1. In description of all plasmids and transcripts the letters $p$ and $t$, respectively, were given at the beginning of their names. The numbering of nucleotides corresponds to the PLRV RNA sequence (accession number X74789). Using the pJF plasmid as a template and sg5end and sg3 primers, a PCR product corresponding to the sgRNA1 was amplified and designated sg (nt 3376-5882). The $\Delta 3^{\prime}$ (nt 3376-5738) PCR product corresponding to sgRNA1 
Table 1. Description of oligonucleotides used in this study.

\begin{tabular}{|c|c|c|c|}
\hline Primer & Sequence $^{\mathrm{a}}\left(5^{\prime}-3^{\prime}\right)$ & Location $^{\mathrm{b}}$ & $\begin{array}{l}\text { PCR template } \\
\text { Construct }^{\mathrm{C}}\end{array}$ \\
\hline $\operatorname{sg} 3$ & ACTACACAACCATGTAAGAGGATCTTGGC & $5854-5882$ & $\begin{array}{l}\mathrm{sg}, \Delta 3^{\prime}, \Delta \mathrm{LS}, \Delta \mathrm{VTE}, \\
\mathrm{mVTE}, \Delta \mathrm{SL}\end{array}$ \\
\hline sg5end & $\begin{array}{l}\text { CCCAAGCTTAATACGACTCACTATAGACAAAAGAACACTGAAGGAGCT- } \\
\text { CAC }\end{array}$ & $3376-3400$ & $\begin{array}{l}\mathrm{sg}, \Delta 3^{\prime}, \Delta \mathrm{RT} \Delta 3^{\prime}, \Delta \mathrm{VTE}, \\
\mathrm{mVTE}, \Delta \mathrm{SL}\end{array}$ \\
\hline T7 $\triangle$ LS_NEW & TTAATACGACTCACTATAGGCGATCAATTGTTAATG & $3574-3590$ & $\Delta \mathrm{LS}, \Delta \mathrm{LS} \Delta 3^{\prime}$ \\
\hline $\operatorname{sg} 3 \Delta \_1$ & TTTCCTCCCTTGGAATGGCTTTTCAGC & $5712-5738$ & $\Delta 3^{\prime}, \Delta \mathrm{LS} \Delta 3^{\prime}$ \\
\hline $\operatorname{sg} 3 \Delta \_2$ & CAGGCTCTGATCCGGAGTCTA & $4215-4236$ & $\Delta \mathrm{RT} \Delta 3^{\prime}$ \\
\hline$\Delta \mathrm{SLU}$ & TACTTCAGTTCGTCAGCGAGGC & 3934-3955 & $\operatorname{psg} \Delta S L$ \\
\hline$\Delta \mathrm{SLD}$ & CATCCTTGAATGCCGGG & 3875-3891 & $\operatorname{psg} \Delta S L$ \\
\hline VTE_L & AGAAACCACGACCGTACTCATTA & $3586-3605$ & psg_mVTE \\
\hline VTE_U & TTAAATGTCAATGGTGGTGTACAA & $3611-3632$ & psg_mVTE \\
\hline RBP_U1 & AATGTCAATGGTGGTGTACAACAA & $3612-3635$ & $\operatorname{psg} \Delta$ VTE \\
\hline RBP_L & CACGACCGTACTCATTAACAATTGA & $3578-3602$ & $\operatorname{psg} \Delta \mathrm{VTE}$ \\
\hline
\end{tabular}

aThe sequence of the T7-promoter is presented in italics, nucleotides not corresponding to the PLRV RNA sequence are in bold. ${ }^{\text {bPosition }}$ in the PLRV genome. 'Names of in vitro transcription PCR-derived templates or names of constructed plasmids with the sequence corresponding to sgRNA1 with modification. Numbering corresponds to PLRV genome (accession number X74789).

with truncated $3^{\prime}$ UTR region was synthesized as above, but using sg5end and sg3 $\Delta_{-} 1$ primers. The $\triangle$ LS (nt 3574-5882) PCR product bearing the last 14 nucleotides of LS was synthesized by PCR with T7 $\triangle$ LS_NEW and sg3 primers. The $\Delta$ LS $\triangle 3^{\prime}$ (nt 35745738) template lacking the $3^{\prime}$ UTR and the LS was obtained with T7 $\Delta$ LS_NEW and sg $3 \Delta \_1$ primer pair. With primers sg5end and sg3 $3 \_2$ and pJF as a template, the $\Delta R T \Delta 3^{\prime}$ (nt 3376-4236) PCR product was synthesized. This template was truncated at position 4236, and does not possess the coding region for the RTD of the viral CP. All PCR products served as templates for in vitro transcripts synthesis that are schematically presented in Fig. 2.

Three psgRNA1 plasmid derivatives, psgmVTE, psg $\triangle$ VTE and psg $\Delta$ SL, bearing modifications within the $\mathrm{CP}$ protein gene were constructed as follows. To delete the $42 \mathrm{nt}$ (3892-3935) corresponding to the stem-loop structure region within the $\mathrm{CP}$ coding sequence (structure not shown), the psgRNA1 template was linearized with $\Delta$ SLD and $\Delta S L U$ primers. To modify the purine-rich region located downstream from the AUG codon of the CP gene, two different constructs were prepared. Using the primer pair VTE_L and VTE_U, purines of the putative regulatory sequence AAAGGAAA (nt 3606-3613) were partially substituted with pyrimidines (TCTTTAAA). Partial (nt 3603-3611) deletion of the purine-rich region was introduced in a PCR reaction with the RBP_L and RBP_U1 primers. Prior to religation and cloning, the PCR products were incubated with $P f u$ polymerase in the presence of nucleotides to generate blunt ends and then phosphorylated with T4 kinase at the $5^{\prime}$ ends. To verify the presence of the desired modifications, DNA of selected clones was sequenced. Derived plasmids psg $\Delta V T E$, psgmVTE and psg $\triangle$ SL served as templates for PCR reactions with the sg5end and sg3 primers. Two more PCR products truncated at nucleotide position 4236 and with deletion or substitution of the purine-rich region were synthesized using sg5end and $\operatorname{sg} 3 \Delta \_2$ primers and psg $\triangle$ VTE and psgmVTE plasmids. These products served as templates for in vitro synthesis of transcripts $\mathrm{t} \Delta \mathrm{VTE}, \mathrm{tmVTE}, \mathrm{t} \Delta \mathrm{SL}, \mathrm{t} \Delta \mathrm{VTE} \Delta \mathrm{RT}$ and tmVTE $\Delta$ RT (Fig. 2).

In vitro transcription. In vitro transcription of PCR generated templates was performed using the RiboMAX Large Scale RNA Production System-T7 (Promega), in the presence of $1 \mathrm{mM} \mathrm{m} 7\left(5^{\prime}\right) \mathrm{Gppp}\left(5^{\prime}\right) \mathrm{G}$ cap analogue (Promega) according to the manufacturer's protocols. After completion of the in vitro transcription reaction, the DNA template was removed by digestion with RQ-DNase (Promega) according to the manufacturer's instructions. To separate capped transcripts from unincorporated $\mathrm{m}^{7} \mathrm{G}-$ pppG (cap-analogue) and ribonucleotides, the mixture was purified on a Sephadex G-50 (Pharmacia) column by centrifugation for $5-8 \mathrm{~min}$ at $1000 \times g$ according to the instructions of the supplier.

Translation in vitro. For in vitro translation 0.08 pmol of each transcript, $5 \mu$ l of WGE (Promega) and $10 \mu \mathrm{Ci}$ of $\left.{ }^{35} \mathrm{~S}\right] \mathrm{L}$-methionine (Amersham) were 

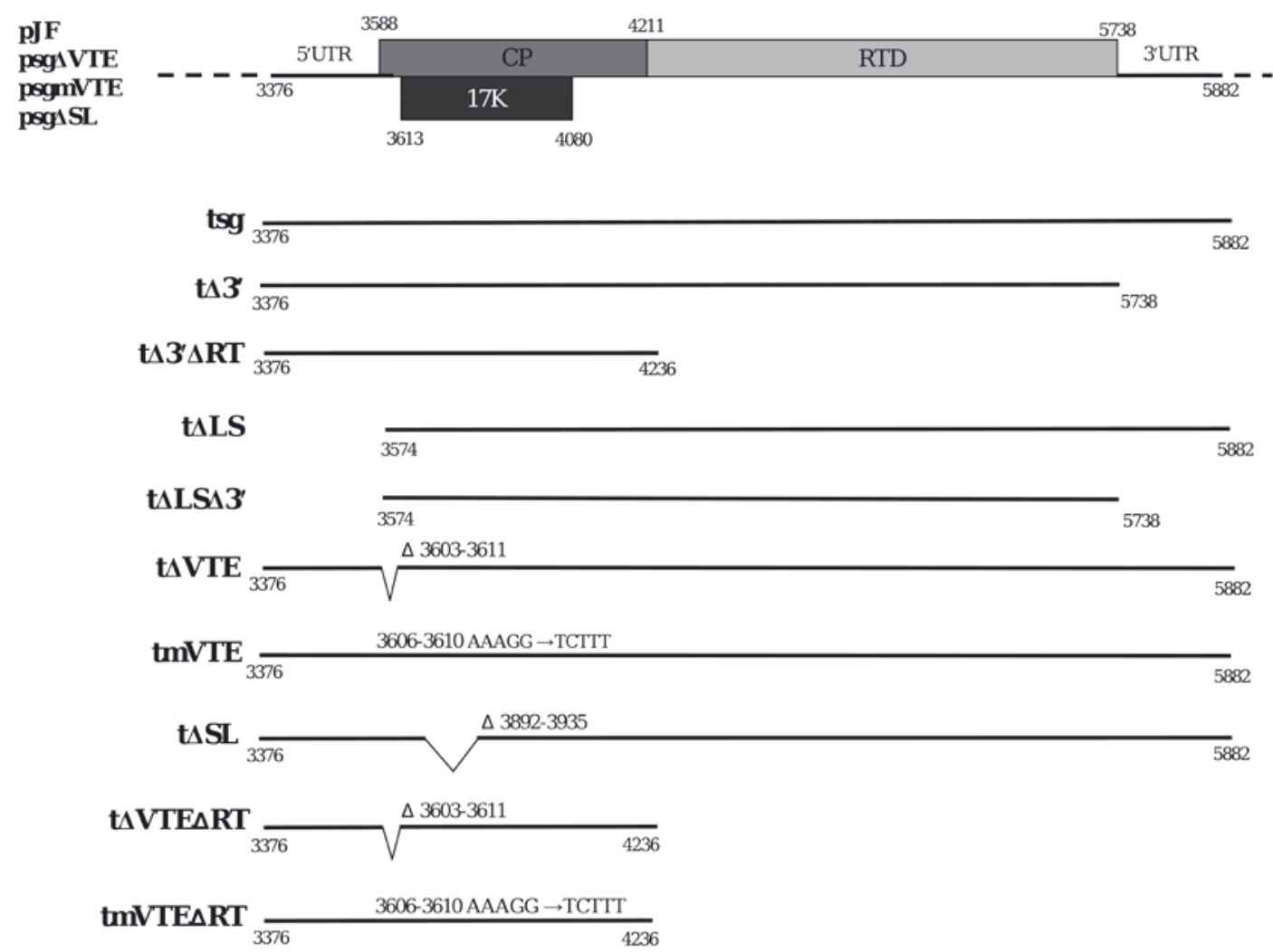

Figure 2. Schematic representation of transcripts used for translation of PLRV sgRNA1 in experiments characterizing the role of coding and non-coding regions.

UTR, untranslated region; CP, coat protein; RTD, read-through domain; 17K, $17 \mathrm{kDa}$ protein. Black lines represent transcripts corresponding to viral RNA. Deletions are indicated by broken line. Numbering of nucleotides according to the PLRV sequence (accession number X74789).

combined in $10 \mu \mathrm{l}$ final volume and incubated at $25^{\circ} \mathrm{C}$ for two $\mathrm{h}$ according to the manufacturer's protocol. The protein products were analyzed by separation on sodium dodecyl sulfate polyacrylamide gel electrophoresis (SDS/PAGE) and subsequent autoradiography.

Quantitative analysis of in vitro synthesized radioactive protein. Bands corresponding to viral $\mathrm{CP}$ and MP were excised from dried SDS/PAGE gels and subjected to radioactivity measurements in scintillation fluid (Orgscynt, BioCare) using a scintillating counter (Pharmacia).

\section{RESULTS AND DISCUSSION}

The role of non-coding sequences of sgRNA1 in the synthesis of $\mathrm{CP}$ and $17 \mathrm{~K}$ proteins

To investigate the role of non-coding regions of sgRNA1 in the translation of the $\mathrm{CP}$ and $17 \mathrm{~K}$ proteins in WGE, transcripts corresponding to sgRNA1 of PLRV but lacking almost the entire $5^{\prime}$ UTR $\left(\Delta\right.$ LS) or having a truncated $3^{\prime}$ UTR $\left(\Delta 3^{\prime}\right)$ were used as templates for in vitro translation re- actions in the presence of $\left[{ }^{35} \mathrm{~S}\right]$ methionine. Translation of both $t \triangle L S$ and $t \Delta 3^{\prime}$ in WGE (Fig. $3 A$, lanes 4 and 2, respectively) yielded two major proteins of $23 \mathrm{kDa}$, corresponding to the $\mathrm{CP}$, and $17 \mathrm{kDa}$, corresponding to the $17 \mathrm{~K}$ protein. The quantification of the radioactivity of the bands excised from the gel showed that the sgRNA 5' UTR has an inhibitory effect on $\mathrm{CP}$ and $17 \mathrm{~K}$ expression since removing most of the subgenomic $5^{\prime}$ UTR resulted in a 2.74-fold increase in translation efficiency (Fig. 3B). This is consistent with previous findings in RRL (Juszczuk et al., 2000); however, the excess of viral proteins produced in the WGE is half that in RRL. Quantification of protein products showed that deletion of the $3^{\prime}$ UTR leads to an increase in the level of translation to 1.2 (Fig. 3B). To test the influence of deletions of both 5' UTR and 3' UTR, translation of $\mathrm{t} \Delta \mathrm{LS} \Delta 3^{\prime}$ was examined. In this case, the level of proteins synthesized was similar to that obtained by translation of $\mathrm{t} \Delta \mathrm{LS}$ (Fig. $3 \mathrm{~A}$, lane 5). The 3' UTR of the PLRV sgRNA1 seems not to exhibit a major regulatory function on the translation of proteins from gRNA1 (Fig. 3A, lane 2) since the amount of viral proteins synthesized from the $t \Delta 3^{\prime}$ templates is slightly higher than that from the sub- 

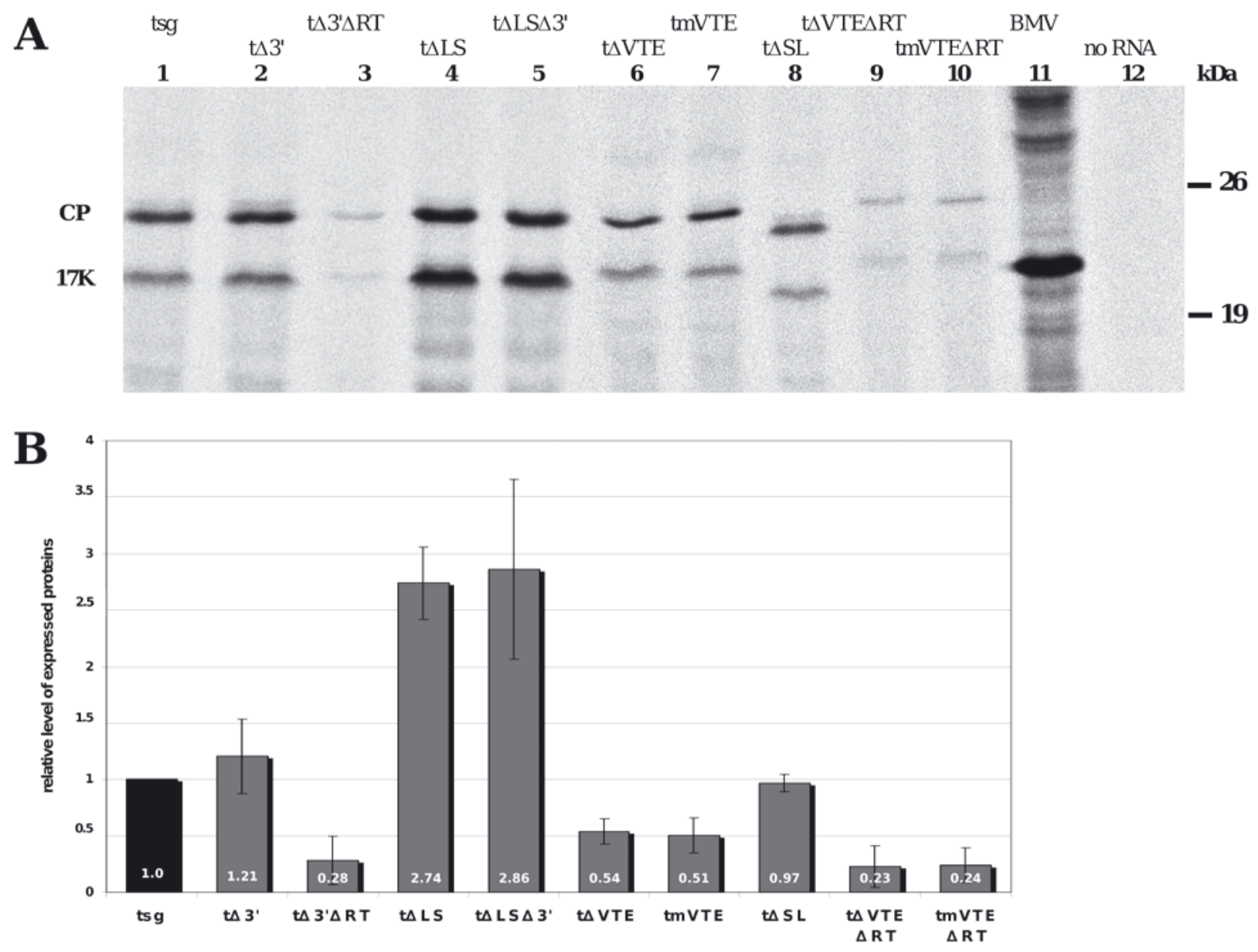

Figure 3. Effect of sequence modifications on translation of sgRNA1 in vitro.

A. Autoradiogram of wheat germ translation products of modified transcripts of PLRV sgRNA1. Lane 1, tsg, transcript corresponding to sgRNA1; lanes 2-5, transcripts with truncated UTRs (Fig. 2); lanes 6-10, transcripts with modifications within the coding sequences of sgRNA1; lane 11, BMV control RNA; lane 12, no RNA. CP, coat protein of PLRV; 17K, $17 \mathrm{kDa}$ protein of PLRV. Molecular mass marker bands are indicated (Fermentas Cat. no. SM0441). B. Quantitative representation of total proteins synthesized in wheat germ extracts from particular transcripts. The diagram represents relative levels of in vitro synthesized proteins. The amount of proteins synthesized from tsg was taken as 1 . The data presented are the average of four independent experiments.

genomic template without modifications (Fig. 3B). Characterizing the influence of the sgRNA1 3' UTR in RRL (Juszczuk et al., 1997) it was suggested that the 3' UTR of PLRV does not influence the translation of the viral $\mathrm{CP}$ and $17 \mathrm{~K}$ proteins. Analyzing the results obtained in WGE we can conclude that the $3^{\prime}$ UTR present in transcripts corresponding to sgRNA1 displays a slight inhibitory effect (Fig. 3B). Our results are in opposition to the finding made for BYDV (PAV), a member of the same family as PLRV but from another genus. In BYDV (PAV) the BTE, located in the 3' UTRs of genomic and subgenomic RNA, was identified (Wang \& Miller, 1995; Wang et al., 1997). This element, together with the 5' UTRs of gRNA and sgRNA1 of BYDV (PAV), plays a significant role in initiation and selective control of cap-independent translation (Wang et al., 1999; Guo et al., 2000).
The influence of coding regions of sgRNA1 on the synthesis of $\mathrm{CP}$ and $17 \mathrm{~K}$ proteins

Coding sequences known to moderate translation of positive-stranded RNA viruses have been identified only for a few viruses. In hepatitis A virus, the coding sequence located downstream of the AUG codon enhances translation of viral gRNA by contributing to the internal ribosome entry site (IRES) function (Graff \& Ehrenfeld, 1998). Translation of Sindbis virus mRNA was also demonstrated to be affected by sequences located downstream of AUG (Frolov \& Schlesinger, 1994). It was demonstrated that hairpin structures are involved in efficient translation and accumulation of Sindbis virus structural proteins (Frolov \& Schlesinger, 1996).

A lack of translation enhancers within the non-coding regions of PLRV sgRNA1 led us to 
search for regulatory elements embedded in the coding sequences. Within the PLRV P1 coding region that is essential for viral multiplication an ORF encoding 5-kDa replication-associated protein 1 (Rap1) has been identified (Jaag et al., 2003). It was also found that Rap1 translation is regulated by an IRES, with an unusual structure and location within ORF1. Core structural elements for this internal ribosome entry include a conserved AUG codon and a downstream GGAGAGAGAGG motif with inverted symmetry. This finding suggests the possible presence of a similar unusual translation regulatory sequence for ORFs expressed from sgRNA1. Sequence analysis of the sgRNA1 revealed the presence of a purine-only region AAAGGAAA (nt 3606-3613) just downstream of the AUG codon of the $\mathrm{CP}$ gene.

Translation of $\mathrm{t} \Delta 3^{\prime} \Delta \mathrm{RT}, \mathrm{t} \Delta \mathrm{VTE}, \mathrm{tmVTE}$ and $\mathrm{t} \triangle \mathrm{SL}$ yielded two major proteins of $23 \mathrm{kDa}$ and 17 kDa (Fig. 3A, lanes 3, 6, 7 and 8, respectively). Quantitative analysis of the proteins synthesized from $\mathrm{t} \triangle \mathrm{SL}$ showed that the amount of $\mathrm{CP}$ and $17 \mathrm{~K}$ proteins is retained at the same level as for tsg (Fig. 3B). This suggests that the stem-loop structure predicted within the $\mathrm{CP}$ gene has no influence on sgRNA1 translation efficiency. In contrast, quantification analyses indicate a strong dependence of the level of sgRNA1 protein expression on the presence of the read-through domain sequence. The absence of almost the entire sequence of ORF5 (nt 4236-5738) reduced the level of sgRNA1 protein synthesis to 0.28 (Fig. 3B). Taking into account the data (Juszczuk et al., 2000) in which deletion of the ORF5 sequence from nucleotides 4457 to 5739 had no influence on the translation level, it can be concluded that the translation regulatory element present within the ORF5 sequence is located between nucleotides 42364457 (numbering on the basis of the PLRV genome).

Changes within $\mathrm{t} \triangle \mathrm{VTE}$ and tmVTE, independently of the type of change, resulted in an about 2fold decrease in translation efficiency. Replacing purines with pyrimidines in tmVTE caused a significant decrease in $\mathrm{CP}$ and $17 \mathrm{~K}$ synthesis. A similar result was obtained by deletion of the six nucleotides AAAGGA (nt 3606-3611) from the purine-only region in $t \triangle V T E$. Given these data, the possibility of the participation of the purine-only sequence in translation regulation cannot be excluded. Deletion of AAAGGA in $\mathrm{t} \triangle \mathrm{VTE}$ dramatically changed the distance between the AUG codons of the $\mathrm{CP}$ and $17 \mathrm{~K}$ genes. Surprisingly, the ratio of the $\mathrm{CP}$ and $17 \mathrm{~K}$ proteins in the case of $\mathrm{t} \triangle \mathrm{VTE}$ translation remained unchanged and was similar to that of tsg (Fig. 3A, lanes 6 and 1, respectively). This observation suggests that the model proposed by Dinesh-Kumar and Miller (1993) to explain the enhancement of translation initiation of the BYDV sgRNA at the CP AUG by pausing of ribosomes at the $17 \mathrm{~K}$ AUG does not agree with what is observed for PLRV CP translation. Translation of $t \triangle V T E \Delta R T$ and tmVTE $\Delta R T$ (Fig. 3A, lanes 9 and 10, respectively) also yielded two major proteins of $23 \mathrm{kDa}$ and $17 \mathrm{kDa}$, but with a nearly 5 -fold decreased efficiency compared to tsg. These results suggest that the regulatory elements within the RTD and purine-only region are independent and may act via unrelated mechanisms.

Our results indicate that translation of PLRV sgRNA1 in WGE is regulated by elements located within the coding sequence of the CP gene and RTD. The most significant effect, nearly 3.6-fold reduction in translation, was observed after removal of the entire coding sequence for the RTD. Taking into account the data obtained by Juszczuk and coworkers (1997) in which deletion of the ORF5 sequence from nucleotides 4457 to 5739 had no influence on the translation level, it can be concluded that the translation regulatory element present within the ORF5 sequence is located between nucleotides 4236-4457 (numbering as for the PLRV genome). The reduction in translation by to one half of that of sgRNA1 in transcripts with modified or partially deleted purine-only region between the AUGs of $\mathrm{CP}$ and $17 \mathrm{~K}$ genes suggests also the presence of an additional element modulating protein expression from sgRNA1 in WGE. This is the first report where the role of coding sequences in translation of a plant RNA virus has been demonstrated, although the mode of action of these elements remains unclear.

\section{Acknowledgements}

Part of this work was carried out with the financial support of the Ministry of Science and Higher Education, Grant No. 2 P04B 00830.

\section{REFERENCES}

Bradrick SS, Walters RW, Gromeier M (2006) The hepatitis $\mathrm{C}$ virus 3'-untranslated region or a poly(A) tract promote efficient translation subsequent to the initiation phase. Nucleic Acids Res 34: 1293-1303.

Danthinne X, Seurinck J, Meulewaeter F, Van Montagu M, Cornelissen M (1993) The 3' untranslated region of satellite tobacco necrosis virus RNA stimulates translation in vitro. Mol Cell Biol 13: 3340-3349.

Dinesh-Kumar SP, Miller WA (1993) Control of start codon choice on a plant viral RNA encoding overlapping genes. Plant Cell 5: 679-692.

Domier LL, McCoppin NK, Larsen RC, D’Arcy CJ (2002) Nucleotide sequence shows that Bean leafroll virus has a Luteovirus-like genome organization. J Gen Virol 83: 1791-1798.

Frolov I, Schlesinger S (1994) Translation of Sindbis virus mRNA: effects of sequences downstream of the initiating codon. J Virol 68: 8111-8117.

Frolov I, Schlesinger S (1996) Translation of Sindbis virus mRNA: analysis of sequences downstream of the ini- 
tiating AUG codon that enhance translation. J Virol 70: 1182-1190.

Gallie DR, Walbot V (1992) Identification of the motifs within the tobacco mosaic virus 5'-leader responsible for enhancing translation. Nucleic Acids Res 20: 46314638.

Graff J, Ehrenfeld E (1998) Coding sequences enhance internal initiation of translation by hepatitis A virus RNA in vitro. J Virol 72: 3571-3577.

Guo L, Allen E, Miller WA (2000) Structure and function of a cap-independent translation element that functions in either the $3^{\prime}$ or the $5^{\prime}$ untranslated region. RNA 6: 1808-1820.

Jaag HM, Kawchuk L, Rohde W, Fischer R, Emans N, Prufer D (2003) An unusual internal ribosomal entry site of inverted symmetry directs expression of a potato leafroll polerovirus replication-associated protein. Proc Natl Acad Sci USA 100: 8939-8944.

Johnston JC, Rochon DM (1996) Both codon context and leader length contribute to efficient expression of two overlapping open reading frames of a cucumber necrosis virus bifunctional subgenomic mRNA. Virology 221: 232-239.

Juszczuk M, Zagórski-Ostoja W, Hulanicka DM (1997) Studies on the translation mechanism of subgenomic RNA of potato leafroll virus. Acta Biochim Polon 44: 69-77.

Juszczuk M, Paczkowska E, Sadowy E, Zagórski W, Hulanicka DM (2000) Effect of genomic and subgenomic leader sequences of potato leafroll virus on gene expression. FEBS Lett 484: 33-36.

Kneller EL, Rakotondrafara AM, Miller WA (2006) Capindependent translation of plant viral RNAs. Virus Res 119: $63-75$.

Mayo MA, Ziegler-Graff V (1996) Molecular biology of luteoviruses. Adv Virus Res 46: 413-460.

Mayo MA, Barker H, Robinson DJ, Tamada T, Harrison BD (1982) Evidence that Potato leafroll virus RNA is positive-stranded, is linked to a small protein and does not contain polyadenylate. J Gen Virol 59: 163-167.

Mayo MA, Robinson DJ, Jolly CA, Hyman L (1989) Nucleotide sequence of potato leafroll luteovirus RNA. J Gen Virol 70: 1037-1051.

Nicolaisen M, Johansen E, Poulsen GB, Borkhardt B (1992) The 5' untranslated region from pea seedborne mosaic potyvirus RNA as a translational enhancer in pea and tobacco protoplasts. FEBS Lett 303: 169-172.
Pooggin MM, Skryabin KG (1992) The 5'-untranslated leader sequence of potato virus X RNA enhances the expression of a heterologous gene in vivo. Mol Gen Genet 234: 329-331.

Qu F, Morris TJ (2000) Cap-independent translational enhancement of turnip crinkle virus genomic and subgenomic RNAs. J Virol 74: 1085-1093.

Sadowy E, Pluta K, Gronenborn B, Hulanicka D (1998) Infectious transcripts from cloned cDNA of potato leafroll luteovirus. Acta Biochim Polon 45: 611-619.

Turner RL, Glynn M, Taylor SC, Cheung MK, Spurr C, Twell D, Foster GD (1999) Analysis of a translational enhancer present within the $5^{\prime}$-terminal sequence of the genomic RNA of potato virus S. Arch Virol 144: 1451-1461.

van der Vossen EA, Neeleman L, Bol JF (1993) Role of the $5^{\prime}$ leader sequence of alfalfa mosaic virus RNA 3 in replication and translation of the viral RNA. Nucleic Acids Res 21: 1361-1367.

Vende P, Piron M, Castagne N, Poncet D (2000) Efficient translation of rotavirus mRNA requires simultaneous interaction of NSP3 with the eukaryotic translation initiation factor eIF4G and the mRNA $3^{\prime}$ end. J Virol 74 : 7064-7071.

Wang S, Miller WA (1995) A sequence located 4.5 to 5 kilobases from the $5^{\prime}$ end of the barley yellow dwarf virus (PAV) genome strongly stimulates translation of uncapped mRNA. J Biol Chem 270: 13446-13452.

Wang S, Browning KS, Miller WA (1997) A viral sequence in the $3^{\prime}$-untranslated region mimics a $5^{\prime}$ cap in facilitating translation of uncapped mRNA. EMBO J 16: 4107-4116.

Wang S, Guo L, Allen E, Miller WA (1999) A potential mechanism for selective control of cap-independent translation by a viral RNA sequence in cis and in trans. RNA 5: 728-738.

Wu B, White KA (1999) A primary determinant of cap-independent translation is located in the 3 '-proximal region of the tomato bushy stunt virus genome. J Virol 73: 8982-8988.

Zelenina DA, Kulaeva OI, Smirnyagina EV, Solovyev AG, Miroshnichenko NA, Fedorkin ON, Rodionova NP, Morozov S, Atabekov JG (1992) Translation enhancing properties of the 5 -leader of potato virus $X$ genomic RNA. FEBS Lett 296: 267-270. 